NOUVELLE

\section{L'adénocarcinome pancréatique : une tumeur dépendante des lipoprotéines}

Sophie Vasseur ${ }^{1-4}$, Fabienne Guillaumond ${ }^{1-4}$
${ }^{1}$ Inserm U1068, Centre de recherche

en cancérologie de Marseille,

F-13288 Marseille, France;

${ }^{2}$ Institut Paoli-Calmettes, F-13009 Marseille, France;

${ }^{3}$ CNRS, UMR7258, F-13009 Marseille, France ;

${ }^{4}$ Université Aix-Marseille, F-13009 Marseille,

France.

sophie.vasseur@inserm.fr

fabienne.marchai@inserm.fr

l'activation constitutive de K-Ras oncogénique, induisent des modifications métaboliques essentielles à l'expansion des cellules tumorales [5]. Définir précisément les mécanismes de reprogrammation métabolique constitue alors un véritable défi pour la découverte de nouvelles stratégies thérapeutiques.

L'avidité des cellules tumorales pancréatiques pour les lipoprotéines en général et le cholestérol en particulier Grâce à I'utilisation des souris PKI décrites ci-dessus, nous avons défini la signature transcriptionnelle de l'adénocarcinome canalaire pancréatique, signature à partir de laquelle une cartographie des voies métaboliques altérées dans cette tumeur a pu être établie. De manière surprenante, les voies impliquées dans le métabolisme lipidique sont les plus activées dans la tumeur, et en particulier celles qui sont associées au catabolisme des lipoprotéines (Figure 1A) [6]. Les lipoprotéines LDL (low-density lipoprotein), principaux réservoirs de cholestérol circulant de l'organisme, sont capturées par les cellules tumorales qui surexpriment à leur surface le récepteur des LDL, le LDLR (LDL receptor). Celui-ci est exprimé par les cellules tumorales pancréatiques quel que soit leur degré de différenciation. Par ailleurs, son expression est très supérieure à celle de l'enzyme limitante de la voie de synthèse du cholestérol, HMGCR (3-hydroxy-3-methylglutarylCoA reductase) [6]. Par conséquent, l'adénocarcinome canalaire pancréatique satisfait ses besoins en cholestérol 
A Reprogrammation lipidique de la tumeur pancréatique

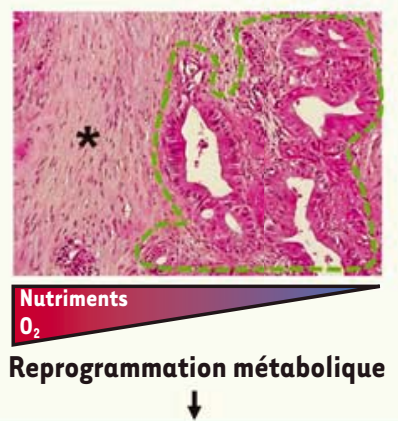

Dérégulation des voies métaboliques lipidiques
B Régulations post-transcriptionnelles du LDLR

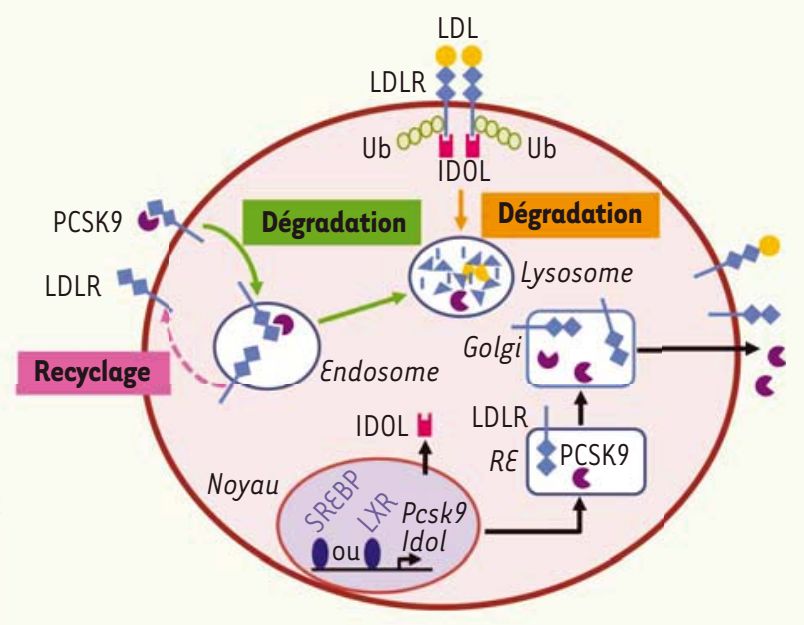

des acides gras
(Ex : Slc27al, Gpam, Pecr)

Catabolisme des acides gras

et acylglycérides

(Ex : Cesld, Ecil, Mgll)

\begin{tabular}{|l|} 
Catabolisme des lipoprotéines \\
(Ex: Ldlr, ApoE, ApoB) \\
Synthèse des leucotriènes \\
(Ex: Alox5, Lta4h, Alox 15) \\
Synthèse des stéroïdes \\
(Ex: Hsdl7b11, Tspo, Ch25h)
\end{tabular}

- Catabolisme des lipoprotéines ( $\varepsilon x:$ Ldlr, ApoE, ApoB)

Synthèse des leucotriènes

Synthèse des stéroïdes

(Ex : Hsd17b11, Tspo, Ch25h)

Figure 1. Adénocarcinome canalaire pancréatique et avidité pour les lipides. A. Reprogrammation lipidique de la tumeur pancréatique. Coloration HPS (hématoxyline, phloxine et safran) d'une coupe de tumeur pancréatique. Les glandes tumorales (délimitées par un trait pointillé vert) sont entourées par une barrière stromale (annotée par *) dense et peu vascularisée qui restreint la diffusion de l' $\mathrm{O}_{2}$ et l'apport en nutriments aux cellules tumorales. En réponse aux stress, hypoxique et nutritif, les cellules tumorales pancréatiques modifient leur activité métabolique pour survivre et proliférer dans cet environnement hostile. Le métabolisme lipidique joue un rôle prépondérant dans cette reprogrammation, comme en témoignent les nombreuses voies altérées dans la tumeur pancréatique par rapport aux pancréas normaux. Ici sont mentionnés quelques exemples de ces voies lipidiques. B. Régulations post-transcriptionnelles du LDLR par IDOL et PCSK9. IDOL, activé transcriptionnellement par le récepteur nucléaire LXR (liver $X$ receptor), induit l'ubiquitination de l'extrémité carboxy-terminale du LDLR puis sa dégradation dans le lysosome. La protéine PCSK9, activée transcriptionnellement par SREBP (sterol regulatory element binding protein), est sécrétée et se lie au domaine extracellulaire du LDLR membranaire. Le complexe LDLR/PCSK9, internalisé par endocytose, empêche le recyclage du LDLR des endosomes vers la membrane plasmique et facilite sa dégradation dans le compartiment lysosomal.

en activant majoritairement la capture du cholestérol exogène au détriment de la voie de synthèse endogène. L'inhibition de la voie de synthèse, par les statines, ne serait donc qu'un coup d'épée dans l'eau pour limiter le contenu en cholestérol des cellules tumorales pancréatiques, face à l'entrée massive de cholestérol via le LDLR. D'ailleurs, aucun bénéfice thérapeutique des statines associées à la chimiothérapie par la gemcitabine ${ }^{l}$ n'a pu être observé chez des patients atteints d'adénocarcinome canalaire pancréatique avancé [7]. Nous avons montré que les tumeurs des patients atteints de ce cancer avec un risque élevé de récurrence tumorale

\footnotetext{
${ }^{1}$ Un antimétabolite analogue de la déoxycytidine.
}

ou métastatique expriment fortement le LDLR, ce qui signe un besoin massif en cholestérol des tumeurs les plus agressives [6].

Quelles sont les causes de cette surexpression du LDLR dans ces tumeurs? Ce récepteur étant finement régulé, des études plus poussées sont nécessaires pour définir les mécanismes moléculaires qui modifient sa stabilité dans la tumeur pancréatique. Différents régulateurs post-transcriptionnels du LDLR ont récemment été découverts: d'une part les protéines ZFP36L1 et ZFP36L2, qui, par leur liaison aux régions non traduites en 3'du LDLR, diminuent sa demi-vie [8], et, d'autre part, les ubiquitine ligases de type ¿3 spécifiques du LDLR, IDOL (inducible degrader of the $L D L R$ ), qui induisent son ubiquitination, puis sa dégradation [9] (Figure 1B). Enfin, les protéines sécrétées PCSK9 (proprotein convertase subtilisin/ kexin 9), en se fixant sur la partie extracellulaire du récepteur, entravent, après I'internalisation de ce dernier, son recyclage de l'endosome vers la membrane plasmique, et favorisent sa dégradation dans le compartiment lysosomal [10] (Figure 1B). Quel que soit le processus à l'origine de l'expression massive du LDLR dans l'adénocarcinome canalaire pancréatique, nous avons démontré que son extinction bouleverse le ratio de cholestérol libre (forme active) et de cholestérol estérifié (forme stockée) dans la cellule tumorale [6]. La surcharge en cholestérol libre résultant de ce déséquilibre est associée à un ralentissement de 
la progression tumorale. De plus, cette perturbation métabolique induite par l'inhibition du LDLR sensibilise les cellules tumorales à la chimiothérapie.

En conclusion, le ciblage du LDLR ouvre donc une nouvelle fenêtre thérapeutique pour combattre l'adénocarcinome canalaire pancréatique. L'identification d'outils thérapeutiques déstabilisant le LDLR, ou bloquant son activité, constitue de réelles options pour le développement de nouveaux essais cliniques. $\diamond$ Pancreatic adenocarcinoma: a tumor highly dependent on lipoproteins

\section{LIENS D'INTÉRÊT}

Les auteurs déclarent n'avoir aucun lien d'intérêt concernant les données publiées dans cet article.

\section{RÉFÉRENCES}

1. Hidalgo M. New insights into pancreatic cancer biology. Ann Oncol 2012 ; 23 (suppl 10) : x135-8.

2. Argiles JM, Busquets $S$, Stemmler B, Lopez-Soriano FJ. Cancer cachexia: understanding the molecular basis. Nat Rev Cancer 2014 ; 14 : 754-62.

3. Aguirre AJ, Bardeesy N, Sinha M, et al. Activated Kras and Ink4a/Arf deficiency cooperate to produce metastatic pancreatic ductal adenocarcinoma. Genes Dev $2003 ; 17$ : 3112-26.

4. Guillaumond F, Leca J, Olivares 0 , et al. Strengthened glycolysis under hypoxia supports tumor symbiosis and hexosamine biosynthesis in pancreatic adenocarcinoma Proc Natl Acad Sci USA 2013 ; 110 : 3919-24.

5. Bryant KL, Mancias JD, Kimmelman AC, Der CJ. KRAS: feeding pancreatic cancer proliferation. Trends Biochem Sci 2014 ; 39 : 91-100.

6. Guillaumond F, Bidaut G, Ouaissi M, et al. Cholesterol uptake disruption, in association with chemotherapy, is a promising combined metabolic therapy for pancreatic adenocarcinoma. Proc Natl Acad Sci USA $2015 ; 112$ : 2473-8.
7. Hong Jy, Nam EM, Lee J, et al. Randomized doubleblinded, placebo-controlled phase II trial of simvastatin and gemcitabine in advanced pancreatic cancer patients. Cancer Chemother Pharmacol 2014 ; $73: 125-30$.

8. Adachi S, Homoto M, Tanaka R, et al. ZFP36LI and ZFP36L2 control LDLR mRNA stability via the ERK-RSK pathway. Nucleic Acids Res 2014 ; 42 : 10037-49.

9. Zelcer N, Hong C, Boyadjian R, Tontonoz P. LXR regulates cholesterol uptake through Idol-dependent ubiquitination of the LDL receptor. Science 2009; $325: 100-4$.

10. Wang $\mathrm{Y}$, Huang $\mathrm{Y}$, Hobbs $\mathrm{HH}$, Cohen JC. Molecular characterization of proprotein convertase subtilisin/ kexin type 9-mediated degradation of the LDLR. J Lipid Res 2012 ; 53 : 1932-43.

11. Bournet $B$, Dufresne $M$, Selves J, et al. Oncogène Kras et cancer du pancréas : trente ans plus tard. Med Sci (Paris) $2013 ; 29: 991-7$

\section{NOUVELLE}

\section{Le VIH- 1 pilote la migration des macrophages}

Christel Vérollet $^{1,2}$, Shanti Souriant ${ }^{1,2}$, Brigitte Raynaud-Messina ${ }^{1,2}$, Isabelle Maridonneau-Parini, ${ }^{1,2}$
${ }^{1}$ CNRS UMR 5089 ; IPBS (Institut de pharmacologie et de biologie structurale), BP64182, 205, route de Narbonne, 31077 Toulouse Cedex 04, France ;

${ }^{2}$ Université de Toulouse ; UPS ; IPBS ; F-31077 Toulouse, France.

isabelle.maridonneau-parini@ipbs.fr verollet@ipbs.fr
Le VIH-1, un virus qui utilise les macrophages pour pénétrer dans les tissus

Le virus de l'immunodéficience humaine (VIH) est responsable du syndrome de l'immunodéficience acquise (Sida). II cible majoritairement les cellules du système immunitaire (lymphocytes T CD4 et monocytes/macrophages), grâce auxquelles il peut disséminer dans l'organisme $[1,2]$. Alors qu'il exerce un effet cytotoxique dans les lymphocytes $T$, il est bien toléré dans les macrophages. Les traitements actuels (trithérapie antivirale) permettent de contrôler la charge virale et de diminuer considérablement la mortalité due à l'infection, mais ils ne permettent pas l'élimination complète du virus. Un des problèmes est la diffi- culté à éliminer certaines cellules infectées, appelées réservoirs viraux [11]. L'existence de ces réservoirs oblige les patients à être traités tout au long de leur vie. Une sous-population minoritaire de lymphocytes T CD4 «mémoire », et les macrophages, font partie de ces réservoirs [3]. Les macrophages infectés présents dans les tissus sont peu accessibles aux traitements, et ont une durée de vie très longue. Leur accumulation dans certains tissus, dont le cerveau, est corrélée à la sévérité de la maladie. Aussi, une stratégie thérapeutique qui inhiberait l'infiltration des macrophages infectés dans les tissus constituerait une avancée majeure. Nous avons donc étudié l'effet du VIH-l sur la migration des macrophages, et mis en évidence (article récemment publié dans Blood [2]) une reprogrammation des capacités migratoires des macrophages infectés.

Le virus reprogramme les capacités migratoires des macrophages et favorise leur infiltration tissulaire L'accumulation de macrophages infectés dans certains tissus pourrait être expliquée par une modification de leurs propriétés migratoires et contribuer ainsi à la dissémination du virus notamment dans des régions difficilement accessibles aux traitements [3]. In vivo, les cellules migrent dans des environnements en deux dimensions (2D) (le long des parois vasculaires par exemple) mais également, et le plus souvent, dans des environnements en 3D. Alors que la 